\title{
Continuous vs. interval exercise training in hypertensive subjects
}

\author{
Kenneth M Madden
}

Hypertension Research (2010) 33, 974; doi:10.1038/hr.2010.119

Correction to: Hypertension Research (2010) 33, 544-545; doi:10.1038/hr.2010.54; published online 30 April 2010

In this article published in the June 2010 issue, the in-text citation for ref. 11 was incorrect. Vlachopoulos et al. ${ }^{11}$ should have read Guimarães et al. ${ }^{11}$ 